\title{
Efeitos da Atenção Dividida no Priming de Repetição
}

\section{Effects of Divided Attention in Repetition Priming}

\author{
Juliana Burges Sbicigo', Gerson Américo Janczura², \\ Jerusa Fumagalli de Salles 3
}

\begin{abstract}
Resumo
O presente artigo tem por objetivo investigar possíveis efeitos da atenção dividida no priming de repetição a partir de uma revisão seletiva da literatura. Foram selecionados estudos realizados com testes de priming perceptual e/ou conceitual, nos quais a divisão da atenção foi realizada na fase de codificação ou de recuperação. Em geral, as evidências indicaram que o priming, tanto o perceptual quanto o conceitual, foi afetado pela atenção dividida na codificação quando a tarefa secundária (ou distratora) foi mais demandante de atenção, exigindo resposta frequente e apresentada sincrônica ao estímulo alvo. Poucos estudos foram realizados na recuperação e eles indicaram imunidade do priming perceptual e conceitual à atenção dividida. Conclui-se que os processos de memória implícita podem exigir recursos atencionais na codificação. Implicações teóricas dos resultados são discutidas.
\end{abstract}

Palavras-chave: Memória implícita. Priming. Atenção. Dual task. Codificação.

\begin{abstract}
This article aims to investigate possible effects of divided attention on repetition priming from a selective review of the literature. Studies were included if they utilized perceptual and/or conceptual priming tasks, in which the division of attention was performed during encoding or retrieval. In general, the results suggested that perceptual and conceptual priming were affected by divided attention during encoding. This effect occurred when the secondary task (or distractor task) demanded higher levels of attention, requiring frequent task responses and it was presented simultaneously to the memory target stimulus. The few studies investigating retrieval showed that perceptual and conceptual priming are not sensitive to divided attention. Therefore, implicit memory processes may require attentional resources in the encoding. Theoretical implications of the results are discussed.
\end{abstract}

Keywords: Implicit memory. Attention. Priming. Dual task. Encoding.

\footnotetext{
${ }^{1}$ Universidade Federal do Rio Grande do Sul, Porto Alegre, Brasil. E-mail: julianasbicigo@gmail.com

${ }^{2}$ Universidade de Brasília, Brasília-DF, Brasil. E-mail: janczura@unb.br

${ }^{3}$ Universidade Federal do Rio Grande do Sul, Porto Alegre, Brasil. E-mail: jerusafs@yahoo.com.br
} 


\section{Introdução}

Lembrar ou recuperar um conteúdo de memória consiste em um processo cognitivo frequente em nosso cotidiano. Além disso, é comum tentarmos realizar duas ou mais tarefas simultaneamente, como lembrar-se de algo durante uma conversação. Sabe-se, porém, que indivíduos não conseguem realizar duas tarefas ao mesmo tempo tão bem como eles a realizariam independentemente (Pashler \& Johnston, 1998). $\mathrm{Na}$ literatura científica, tem sido pesquisado se a distração pode afetar a memória implícita, que consiste na recuperação não consciente e não intencional de experiências recentes, tipicamente avaliada com medidas de priming (Graf \& Schacter, 1985; Mulligan, 2003). Dentre os diferentes paradigmas de priming (ex.: associativo), os efeitos da distração têm sido investigados, sobretudo, em testes de priming de repetição. Nesses testes, a memória implícita é demonstrada por meio de uma facilitação na habilidade de processar um estímulo como resultado de um encontro recente com aquele estímulo ou um estímulo relacionado sem que haja consciência dessa relação (Graf \& Schacter, 1985; Schacter, 1987). O encontro prévio facilita o processamento do item, o que significa que ele deverá ser recuperado de forma automática (Jacoby, 1991).

Estudos sobre uma possível participação da atenção nos processos de memória implícita tornaram-se mais frequentes em meados da década de 1990. Até esse período, os efeitos da distração foram estudados na memória explícita (Mulligan, 2003). Nacionalmente, há pesquisas no campo da memória implícita (ex.: Salles, Machado \& Janczura, 2011), mas não são encontradas investigações que explorem o papel da atenção nesse tipo de memória ou artigos teóricos publicados em português.

Os resultados dos estudos internacionais iniciados em meados da década de 1990 consolidaram a visão de que a memória implícita (priming) envolve processos automáticos, isto é, que não envolvem a atenção (Isingrini, Vazou \& Leroy, 1995; Logan, 1990; Schacter, 1987; Tulving \& Schacter, 1990), visão amplamente difundida na Psicologia Cognitiva até os dias atuais. Por outro lado, os estudos experimentais que avaliaram os efeitos da atenção dividida na memória implícita não têm confirmado essa visão de forma unânime, o que torna relevante revisar as evidências disponíveis sobre o tema.

Tradicionalmente, priming de repetição e automaticidade têm sido considerados como resultado de um mecanismo comum de armazenamento e recuperação de representações individuais de exposição a um estímulo específico (Logan, 1990). Quando um estímulo aparece pela segunda vez no priming, há uma redução das demandas atencionais de processamento daquele estímulo se comparado a estímulos novos. A redução de recursos atencionais deve tornar automática a recuperação de informações daquele estímulo se comparado a estímulos novos (Logan, 1990; Jacoby, 1991; Stevens et al., 2007). Considerar os processos de memória implícita (priming) como automáticos significa afirmar que eles devem ser eficazes mesmo na presença de estímulos distratores, seja no momento de exposição à informação (codificação), seja no momento de recuperá-la, em testes perceptuais ou conceituais.

Pesquisas avaliando o papel da atenção no priming têm sido guiadas, sobretudo, pela 
perspectiva da Teoria de Transferência Apropriada de Processamento (TransferAppropriate Processing - TAP, Roediger, 1990). A TAP divide os processos envolvidos nos testes de memória como perceptuais e conceituais. O processamento dirigido perceptualmente enfatiza as características superficiais (físicas [formas, cor, posição, etc.]) dos estímulos, sendo sensíveis a manipulações de variáveis perceptuais (visual vs. aditivo), mas não a manipulações conceituais. $\mathrm{O}$ processamento dirigido conceitualmente enfatiza a análise semântica dos estímulos (Roediger, 1990) e é afetado por manipulações da codificação conceitual, tais como manipulação de geração de itens (Blaxton, 1992) e de níveis de processamento (Hamann,1990). Por dependerem do acesso ao significado, essa teoria afirma que as tarefas de priming de repetição conceitual são consideradas mais demandantes de atenção que as tarefas perceptuais (Roediger, 1990).

Alternativamente, uma terceira perspectiva, desenvolvida por Mulligan (2003), é baseada no modelo de "gargalo" ou "filtro" central (central bottleneck) de Pashler (1994). Ela supõe que qualquer tarefa de priming de repetição (perceptual ou conceitual) pode envolver a participação da atenção desde que a tarefa secundária ou distratora exija respostas frequentes e seja apresentada ao mesmo tempo em que o alvo de priming. Nesse caso, selecionar uma resposta prejudicaria a codificação do alvo e exigiria processos atencionais.

Em estudos experimentais de laboratório, o papel da atenção na memória implícita tem sido testado usando o paradigma da atenção dividida (AD), no qual o teste principal (ex.: priming) é realizado simultaneamente a outra tarefa (ex.: monitorar sílabas), denominada de tarefa secundária (TS) ou distratora. Para comparação, há uma condição de atenção total na qual o teste principal é realizado isoladamente. A predição é de que se TS não exerce efeito sobre o desempenho na tarefa de memória, infere-se que o processo de memória é automático (implícito ou não consciente). Ao contrário, se a TS exerce efeito sobre o desempenho, julga-se que o processo tem participação da atenção e, portanto, é controlado (Craik et al., 1996).

Desse modo, o objetivo deste estudo foi investigar possíveis efeitos da $\mathrm{AD}$ no priming de repetição a partir de uma revisão seletiva da literatura. Mais especificamente, buscou-se verificar os efeitos da $\mathrm{AD}$ em testes de priming de repetição perceptual e conceitual nas fases de codificação e recuperação da memória implícita.

\section{Método}

A revisão seletiva foi baseada na busca de artigos obtidos utilizando dois mecanismos: (i) busca manual em revistas reconhecidas por publicar estudos experimentais na área da memória (Journal of Experimental Psychology: Learning, Memory and Cognition; Memory and Cognition; Memory; Journal of Memory and Language; European Journal of Cognitive Psychology) e (ii) busca adicional nas bases de dados Web Of Science e PsycInfo até 2014, utilizando a combinação entre os termos "implicit memory AND divided attention", "priming AND divided attention", "repetition priming AND divided attention". Para atender ao objetivo proposto, foram incluídos na presente revisão: a) estudos em que foram utilizados testes de priming de repetição, de modo que aqueles com priming associativo e priming semântico foram descartados; b) estudos nos quais os testes 
ajustam-se na classificação perceptual ou conceitual (conforme a teoria da TAP, Roediger, 1990); c) estudos conduzidos com amostra não clínica e d) estudos com testes de priming de repetição em que o efeito da atenção foi investigado com manipulações da atenção dividida, de modo que estudos utilizando manipulações da atenção seletiva foram excluídos. Após a aplicação dos critérios de inclusão, o mecanismo de busca (i) elegeu 23 artigos dos seguintes periódicos: Journal of Experimental Psychology: Learning, Memory and Cognition (6); Memory and Cognition (8); Memory (3); Journal of Memory and Language (2); European Journal of Cognitive Psychology (4). O mecanismo (ii) adicionou oito artigos das bases de dados Web of Science e PsycInfo que preencheram os critérios de inclusão após eliminação de artigos repetidos entre as bases e em relação ao mecanismo de busca (i).

\section{Resultados}

A partir da busca de estudos, verificou-se que, enquanto há uma tradição de estudos analisando os efeitos da $\mathrm{AD}$ na codificação implícita $(n=29)$, há ainda uma escassez de pesquisas realizadas na fase de recuperação $(n=$ 2). A Tabela 1 sumariza os estudos identificados, informando o teste de priming, a tarefa secundária (TS) e se o priming foi imune ou afetado pela AD. Nos 29 artigos voltados à codificação, a AD afetou o priming de repetição em 20 experimentos e o priming foi imune ao seu efeito em 33 experimentos. Nos dois artigos voltados à fase de recuperação, priming foi imune ao efeito da AD. A descrição dos resultados foi organizada em dois tópicos: $\mathrm{AD}$ na codificação implícita e $\mathrm{AD}$ na recuperação implícita, ambos abordando testes perceptuais e conceituais.

Tabela 1. Estudos de Priming de Repetição com Atenção Dividida na Codificação e Recuperação

\begin{tabular}{|c|c|c|c|}
\hline Autores & Testes de Priming & Tarefa Secundária (TS) & $\begin{array}{l}\text { Imune } v s . \\
\text { afetado }\end{array}$ \\
\hline \multicolumn{4}{|c|}{ Atenção dividida na codificação } \\
\hline $\begin{array}{l}\text { Priming Perceptual } \\
\text { Mulligan \& Hornstein } \\
(2000) \text {, exp.1-4 }\end{array}$ & Identificação perceptual & Tarefa Stroop & Afetada \\
\hline $\begin{array}{l}\text { Mulligan (2002), } \\
\text { exp.1-2 }\end{array}$ & Identificação perceptual & Tarefa Stroop & $\begin{array}{l}\text { Imune (exp.1) } \\
\text { Afetada (exp.2) }\end{array}$ \\
\hline $\begin{array}{l}\text { Mulligan (2003), } \\
\text { exp.1-4 }\end{array}$ & Identificação perceptual & $\begin{array}{l}\text { Monitoramento de dígito } \\
\text { Decisão par ou ímpar } \\
\text { Monitoramento de tom }\end{array}$ & $\begin{array}{l}\text { Imune (exp.1) } \\
\text { Afetada (exp. } 2 \text {-3) }\end{array}$ \\
\hline $\begin{array}{l}\text { Mulligan et al. } \\
(2007) \text {, exp.1-2 }\end{array}$ & Identificação perceptual & $\begin{array}{l}\text { Monitoramento de dígito } \\
\text { Decisão par ou ímpar }\end{array}$ & $\begin{array}{l}\text { Imune (exp.1) } \\
\text { Afetada (exp.2) }\end{array}$ \\
\hline $\begin{array}{l}\text { Mulligan \& Peterson } \\
\text { (2008), exp.6 }\end{array}$ & Identificação perceptual & Decisão par ou ímpar & Afetada \\
\hline $\begin{array}{l}\text { Schmitter-Edgecombe } \\
\text { (1996a) }\end{array}$ & $\begin{array}{l}\text { Identificação } \\
\text { taleidoscópica }\end{array}$ & Decisão de tom & Imune \\
\hline $\begin{array}{l}\text { Schmitter-Edgecombe } \\
\text { (1996b) }\end{array}$ & $\begin{array}{l}\text { Identificação } \\
\text { taleidoscópica }\end{array}$ & Decisão de tom & Imune \\
\hline $\begin{array}{l}\text { Schmitter-Edgecombe } \\
\text { (1996a) }\end{array}$ & Clarificação perceptual & Decisão de tom & Imune \\
\hline Smith \& Oscar-Berman & Decisão Lexical & Monitoramento de barras & Imune \\
\hline
\end{tabular}
(1990) 


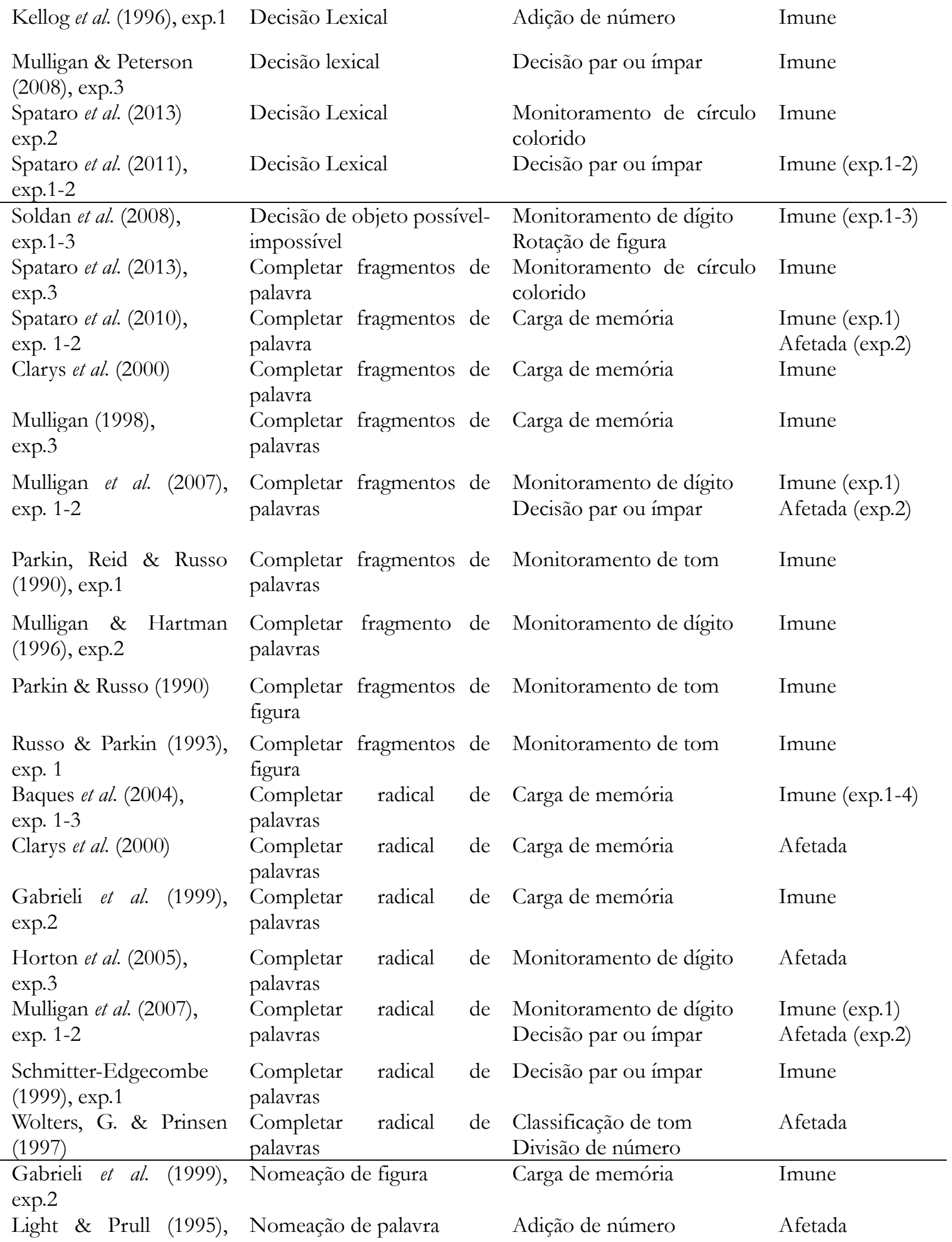
exp.1-2

\section{Priming Conceitual}

\begin{tabular}{|c|c|c|c|}
\hline $\begin{array}{l}\text { Mulligan \& Peterson } \\
\text { (2008), exp.1 }\end{array}$ & Verificação de categoria & Decisão par ou ímpar & Imune \\
\hline $\begin{array}{l}\text { Light et al. (2000), } \\
\text { exp.2-3 }\end{array}$ & Verificação de categoria & Adição de número & Imune \\
\hline $\begin{array}{l}\text { Gabrieli et al. (1999), } \\
\text { exp.4 }\end{array}$ & Verificação de categoria & Monitoramento de dígito & Imune \\
\hline
\end{tabular}


Spataro et al. (2013), exp.4

Isingrini et al. (1995)

Mulligan \& Hartman (1996), exp.1

Schmitter-Edgecombe (1996)

Mulligan (1997)

Gabrieli et al. (1999), exp.4

Mulligan \& Stone (1999), exp.1-4

Schmitter-Edgecombe (1999), exp.2

Light \& Prull (2000), exp.1

Parker et al. (2012)

Prull (2013), exp.1, 3, 5
Verificação de categoria

Produção de exemplar de categoria

Produção de exemplar de categoria

Produção de exemplar de categoria

Produção de exemplar de categoria

Produção de exemplar de categoria

Produção de exemplar de Monitoramento de dígito categoria

Produção de exemplar de categoria

Produção de exemplar de categoria

Produção de exemplar de categoria

Geração verbal

Monitoramento

consoante

Monitoramento de dígito

Decisão de tom

Carga de memória

Monitoramento de dígito
Monitoramento de círculo

Mulligan (1998), exp.1

Conhecimento geral

Atenção dividida na recuperação

Lozito \& Mulligan Identificação perceptual (2010), exp. 1

Clarke \& Butler (2008), exp. 1,2

Completar

radical palavra

Lozito \& Mulligan

(2010), exp. 2

Completar radical palavra

Lozito \& Mulligan, Produção de exemplar de (2010), exp. 3
Decisão par ou ímpar

Adição de número

Carga de memória

Decisão par ou ímpar Adição de número Monitoramento de dígito
Monitoramento de sílabas
Monitoramento de dígitos
Decisão par ou ímpar
Decisão uma/duas sílabas

Imune

Imune (exp.1a)

Afetado (exp.1b-

4)

Afetado

Afetado

Imune

Afetado

Afetado
Afetado

Afetado

de Monitoramento de sílabas

Imune

Imune

Monitoramento de dígitos

Decisão par ou ímpar

Decisão uma/duas sílabas

Monitoramento de sílabas

Monitoramento de dígitos

Decisão par ou ímpar

Decisão uma/duas sílabas

Fonte: Elaborada pelos autores.

\section{AD na codificação implícita}

\section{Priming perceptual}

Inicialmente, Parkin e Russo (1990)

verificaram imunidade do priming ao efeito da $\mathrm{AD}$.

Os participantes foram expostos a figuras de objetos comuns de forma fragmentada até que eles identificassem o objeto. $\mathrm{Na}$ condição de AD, esse teste foi realizado com uma TS de monitoramento de tons, que consistiu em classificar sequências de tons puros (em Hz) em alto, médio e baixo. Após 24 horas, os participantes identificaram figuras fragmentadas 
(metade das figuras vistas antes) no teste implícito (tal como na seção de estudo). O efeito de priming consistiu na identificação de figuras estudadas em níveis de identificação mais baixos (com menos informação) se comparado às figuras não estudadas (novas / linha de base).

A imunidade do priming à $\mathrm{AD}$, observada em Parkin e Russo (1990), foi reproduzida em estudos posteriores com diferentes testes de memória implícita (ex.: Clarys, 2000; Mulligan \& Hartman, 1996; Mulligan, 1998; Russo \& Parkin, 1993). Por outro lado, houve redução do priming em alguns estudos (ex.: Light \& Prull, 1995; Mulligan \& Hornstein, 2000; Mulligan, 2002; Wolters, \& Prinsen, 1997).

No estudo de Mulligan (2003), foram realizados três experimentos, no qual foram utilizadas TS adaptadas da tarefa de monitoramento de dígitos. No experimento 4 , as TS foram baseadas em formas geométricas ou em tons puros. A codificação foi ler os alvos em voz alta para que eles fossem obrigatoriamente processados quanto às suas propriedades. Os resultados indicaram que o priming perceptual a) foi imune à $\mathrm{AD}$ quando a TS foi auditiva (intermodal), apresentada de forma não simultânea ao alvo e exigiu respostas infrequentes (exp.1), replicando outros estudos (ex.: Gabrieli et al., 1999); b) foi afetado tanto com TS intra quanto intermodal, ambas apresentadas simultaneamente ao alvo (exp.2); e c) foi afetado quando a frequência de resposta da TS aumentou independente da sincronia com a apresentação do alvo (exp.3). Esses resultados foram generalizados quando foi utilizada uma TS visual (intramodal) baseada em formas geométricas (flankers) e em tons puros (intermodal), ambas apresentadas com o alvo e com respostas frequentes (exp.4). Assim, os efeitos encontrados nos experimentos anteriores não foram resultado da combinação específica entre os testes de memória e TS utilizadas.

Mulligan (2003) interpretou seus resultados à luz do que ele chamou de hipótese de seleção do distrator, resolvendo a inconsistência dos resultados de estudos prévios. Baseada no modelo de "gargalo" ou "filtro" central (central bottleneck) de Pashler (1994), a hipótese prediz que a seleção de resposta à TS prejudica a codificação da memória. Especificamente, quando a TS exige respostas frequentes e quando ela é apresentada ao mesmo tempo em que o alvo, o processo de selecionar uma resposta provavelmente prejudica a codificação do alvo. Nos experimentos de Mulligan (2003), houve apoio a essa hipótese, uma vez que o desempenho no priming foi reduzido à medida que foi aumentada a frequência de resposta à TS (ou distratora), independente da sincronia com o alvo. A redução ocorreu tanto em TS intra quanto intermodal. Resultados similares foram observados em outros testes perceptuais elaborados na modalidade auditiva, como em identificação perceptual e completar fragmentos de palavras, com TS visual de decisão par ou ímpar (Mulligan, Duke \& Cooper, 2007).

A hipótese de seleção do distrator também explicou a imunidade do priming na decisão de objeto possível/impossível, com a TS de monitoramento de dígitos (resposta infrequente, apresentação não sincrônica ao alvo de memória), porém não se ajustou à imunidade do priming observada quando foi utilizada a TS de rotação de figura - sincrônica ao alvo de memória e com resposta frequente (Soldan, Mangels \& Cooper, 2008). Outros resultados inconsistentes 
com a referida hipótese foram: a) imunidade do priming em completar radical de palavra, com uma TS de carga de memória - resposta frequente (Baques, Sáiz \& Bowers, 2004); b) redução do priming no mesmo teste do item a, mas com a TS de monitoramento de dígitos - baixa frequência de resposta (Horton, Wilson, Vonk, Kirby \& Nielsen, 2005), e c) imunidade do priming em decisão lexical, com a TS de decisão par ou ímpar - resposta frequente (Mulligan \& Perterson, 2008) e adição de número (Kellog, Newcombe, Kammer \& Schmitt, 1996).

Estudos mais recentes (Spataro, RossiArnaud \& Pazzano, 2010; Spataro, Mulligan \& Rossi-Arnaud, 2011, 2013) expandiram as evidências no âmbito do priming perceptual, já que eles investigaram as condições nas quais a $\mathrm{AD}$ poderia afetar completar fragmentos de palavras e decisão lexical. Spataro et al. (2010) analisaram o teste de completar fragmentos de palavras com única (apenas uma resposta ao radical) e múltiplas soluções (várias respostas emitidas para completar o radical). A TS foi uma carga de memória de curto prazo (como em Clarys et al., 2000; Mulligan, 1998), com sequências de sete itens de letras e números, apresentadas antes do alvo, e que deveriam ser recordadas segundos depois da aparição do alvo. Priming foi afetado na condição de AD com tempo de codificação mais curto, mas foi imune quando a apresentação de itens de AD e atenção total foi mista (em vez de serem mostrados em condições separadas). Ainda, os efeitos não se diferenciaram entre as condições de única e múltiplas soluções (nesse último caso, a hipótese é de que são eliciadas respostas competidoras que tornariam o priming vulnerável à divisão de atenção). Resultado similar foi obtido com decisão lexical, com TS de decisão par ou ímpar, quando ela foi manipulada para ativar única ou múltiplas representações (Spataro et al., 2011).

Um resultado surpreendente foi documentado por Spataro et al. (2013) em completar fragmentos de palavra: o efeito de priming foi "maior" na condição de $\mathrm{AD}$ se comparado à condição de atenção total. Tal fenômeno, cunhado pelos autores de attentional boost effect, ocorreu com uma TS intramodal, porém com resposta menos frequente. $\mathrm{Na}$ fase de estudo, os estímulos dos testes de completar fragmentos de palavra e decisão lexical foram lidos em voz alta pelos participantes enquanto monitoravam a cor de um pequeno círculo abaixo da palavra. A instrução foi para pressionarem a barra de espaço quando vissem um círculo vermelho entre os círculos verdes mais frequentes. Palavras acompanhadas pelo estímulo alvo da TS (círculo vermelho) produziram maior priming perceptual do que palavras acompanhadas pelos círculos verdes. Ainda, priming foi maior na condição de $\mathrm{AD}$ se comparado à condição de atenção total. Por outro lado, a tarefa conceitual de classificação semântica não foi beneficiada pela atenção.

Em geral, os estudos apresentados na Tabela 1 indicam que o priming perceptual envolve alguma quantidade de recursos atencionais. Isso foi verificado em identificação perceptual, completar fragmentos de palavras, completar radical de palavras e nomeação de palavras, sobretudo quando foram realizadas com TS mais demandantes de atenção (resposta frequente e sincrônicas ao alvo de memória). Por outro lado, decisão lexical se mostrou resistente à $\mathrm{AD}$, o que sugere que o tipo de teste de priming, e não só as 
características da TS, explicariam o papel da atenção na recuperação implícita.

\section{Priming conceitual}

Em geral, o efeito de priming em testes conceituais foi afetado pela AD (Gabrieli et al., 1999; Light et al., 2000; Mulligan, 1997, 1998; Mulligan \& Hartman, 1996; Mulligan \& Stone, 1999; Prull et al., 2013; Schmitter-Edgecombe, 1999), embora tenha sido imune algumas vezes (Isingrini et al., 1995; Mulligan, 1997; Mulligan \& Stone, 1999). Mulligan (1997) avançou nessa questão, variando a carga atencional da TS em 0 (condição de atenção total), 1 (carga baixa), 3 (carga média) e 5 (carga alta) sequências de letras e números, apresentados antes do estímulo de estudo e que deveriam ser recordadas poucos segundos depois. Priming foi eliminado na condição de carga alta, mas não houve diferença entre as outras condições de menor carga, corroborando os resultados de Isingrini et al. (1995).

Gabrieli et al. (1999) identificou que nem todos os testes conceituais são afetados pela AD. $\mathrm{Na}$ fase de estudo, os participantes foram expostos a uma série de exemplos de categorias e no teste realizaram produção de exemplar da categoria ou verificação de categoria. $\mathrm{Na}$ atenção total, cada palavra foi julgada como produzida pelo homem ou encontrada na natureza. $\mathrm{Na} A D$, além desse julgamento, era realizada a TS de monitoramento de dígito (baixa frequência de resposta). Em verificação de categoria, foram apresentadas questões de verificação (ex.: É um animal selvagem?), seguidas pelo item (tigre) que poderia ou não ser membro da categoria. Em produção de exemplar de categoria, foram mostrados nomes de categorias e os participantes deveriam gerar um exemplar que viesse à mente. Priming foi reduzido em produção de exemplar da categoria, como em estudos anteriores (ex.: Mulligan \& Hartman, 1996; Mulligan, 1997), mas verificação da categoria foi inalterada. Esse achado foi replicado em outros estudos (Light et al., 2000; Mulligan \& Peterson, 2008). Light et al. (2000), por exemplo, utilizaram uma TS na qual era necessário relatar se a soma dos números apresentados com o exemplar da categoria era par ou ímpar (TS mais demandante de atenção). Enquanto a tarefa de verificação da categoria foi imune aos efeitos da atenção, o priming em produção de exemplar da categoria foi afetado. Spataro et al. (2013), utilizando a TS de monitoramento de círculo, não identificaram qualquer efeito da $\mathrm{AD}$ no priming em verificação de categoria. Os autores sugeriram que o teste não deve refletir processamento conceitual e pode ser classificado como sendo um teste de identificação.

Para Parker et al. (2012), os efeitos de AD, encontrados no teste de produção de exemplar da categoria em Gabrieli et al. (1999), ocorreram porque a codificação realizada (dizer se o exemplar era produzido pelo homem ou encontrado na natureza) provavelmente desviou a atenção do processamento de relações entre categorias e exemplares. Em Parker et al. (2012), os participantes foram expostos a pares de palavras e a codificação foi realizada com uma das duas tarefas: (i) expressar o grau de associação entre as palavras ou (ii) julgar se o exemplar pertencia à categoria (nomes de categorias foram apresentados com um possível exemplar; metade dos pares foram congruentes). Esses testes foram realizados com uma TS de carga de memória 
(cargas de 0, 3, 5 e 7 itens) composta por sequências de letras e números, como em Mulligan (1997). Enquanto a AD reduziu a recordação de categoria com pista nas duas condições de codificação, o desempenho em produção de exemplar da categoria foi inalterado entre as condições de atenção quando a codificação foi o julgamento de categoria. A conclusão foi de que quando são realizadas associações entre categoria-exemplar na codificação priming é inalterado.

Prull (2013) avaliou outro teste de priming conceitual: geração verbal. Nesse teste, os participantes codificam nomes acidentalmente por gerar um verbo apropriado para um nome (ex.: caneta - escrever). No teste, a tarefa de geração verbal é repetida com nomes codificados misturados com novos nomes (ex.: Seger, Rabin, Desmond \& Gabrieli, 1999). Quando a TS foi menos demandante de atenção (classificação de dígito par ou ímpar), priming foi afetado fracamente, enquanto essa manipulação afetou reconhecimento. Por outro lado, quando a TS foi mais demandante de atenção (aritmética mental: indicar se a soma dos números aparecendo com o alvo era par ou ímpar, como em Light et al., 2000), priming foi afetado (redução de 30\% comparado com a condição de atenção total).

Prull (2013) buscou ainda minimizar as contribuições ao priming de outros processos que não fossem próprios da seleção verbal na tarefa de geração verbal. Assim, foi realizada uma nova condição de AD: os participantes deveriam pensar em uma ação apropriada para cada nome, enquanto repetiam uma sílaba em voz alta (ex.: dadada) em uma taxa de duas sílabas por segundo e respondiam à TS de soma de dígitos. O prejuízo no priming foi ainda maior nesse experimento (59\% em relação à condição de atenção total).

Em síntese, a $\mathrm{AD}$ com TS menos demandantes de atenção (baixa frequência de resposta), ainda que sincrônicas ao alvo de memória, tanto afetaram (ex.: Gabrieli et al., 1999; Mulligan, 1998) quanto não afetaram o priming (ex.: Isingrini et al., 1995; Mulligan \& Stone, 1999). As TS mais demandantes de atenção (alta frequência de resposta e sincrônicas ao alvo de memória) produziram efeitos consistentes de redução no priming conceitual (ex.: Mulligan, 1997; Prull, 2013), apesar de verificação de categoria e decisão lexical terem sido resistentes às TS.

\section{$\mathrm{AD}$ na recuperação implícita}

\section{Priming perceptual e conceitual}

Apenas dois estudos (Clarke \& Butler, 2008; Lozito \& Mulligan, 2010) avaliaram os efeitos da AD na fase de recuperação. Em Clarke e Butler (2008), o desempenho na tarefa de completar radical de palavras foi imune ao efeito da $\mathrm{AD}$, utilizando uma tarefa de monitoramento de sílabas (monitorar sequências de três palavras de duas sílabas), enquanto recordação com pista foi afetada. Para ampliar o resultado, foi utilizada uma versão mais difícil de completar radical de palavra, na qual os estímulos foram apresentados de forma rápida (33ms vs. $23 \mathrm{~ms}$ ) na fase de recuperação.

Os achados de Clarke e Butler (2008) foram ampliados por Lozito e Mulligan (2010). Eles aplicaram duas tarefas perceptuais (identificação perceptual e completar radical de palavra) e uma conceitual (produção de exemplar 
da categoria). Foram utilizados diferentes tipos de TS - verbais (monitoramento de sílaba, decisão uma ou duas sílabas) e numéricas (monitoramento de dígito, decisão de número par ou ímpar) -, assim como foi manipulada a frequência de resposta (alta vs. baixa frequência). $\mathrm{O}$ desempenho no priming foi imune à $\mathrm{AD}$, independentemente do tipo de tarefa de memória, de TS e do tipo de resposta. Contudo, uma tendência numérica à redução do priming ocorreu somente quando as TS usaram o mesmo material (verbal) da tarefa de memória. Os autores também calcularam os efeitos de realizar a tarefa de memória sobre o desempenho na TS, conforme recomendado por Craik et al. (1996). Esse cálculo indicou que a recuperação implícita em itens estudados geralmente facilitou $O$ desempenho na TS (Lozito \& Mulligan, 2010). Isso reforça o quanto o teste de memória exigiu poucos recursos da atenção.

\section{Discussão}

O objetivo da presente revisão foi investigar possíveis efeitos da AD no priming de repetição perceptual e conceitual a partir de uma revisão seletiva da literatura. As evidências revisadas indicaram que a $\mathrm{AD}$ pode afetar priming perceptual e conceitual na codificação (p.ex.: Gabrieli et al., 1999; Mulligan \& Hartaman, 1996; Mulligan, 2003; Spataro et al., 2013). Esse efeito ocorre dependendo do tipo de teste implícito e das características da TS. Esse último fator parece ter maior contribuição, já que testes de priming perceptual foram imunes à $\mathrm{AD}$ quando a TS foi menos demandante de atenção (com resposta menos frequente e/ou não foi apresentada sincrônica ao alvo, ex.: Clarys et al., 2000; Isingrini et al., 1995; Mulligan \& Hartman, 1996; Mulligan, 2003; Soldan et al., 2008), mas foi afetado quando foram utilizadas TS mais demandantes (com resposta frequente e apresentadas sincrônicas ao alvo; ex.: Clarys et al., 2000; Gabrieli et al., 1999; Ganor-Stern et al., 1998; Horton et al., 2005; Light \& Prull, 1995; Mulligan, 2003; Spataro et al., 2010; Wolters \& Prinsen, 1997).

No priming conceitual, o desempenho também foi afetado pela $\mathrm{AD}$ nas condições de tarefas mais demandantes de atenção (Gabrieli et al., 1999; Light et al., 2000; Mulligan, 1997, 1998; Mulligan \& Hartman, 1996; Prull, 2013; Schmitter-Edgecombe, 1999). Em relação às tarefas menos demandantes, as evidências são controversas, já que priming conceitual foi tanto inalterado (Isingrini et al., 1995; Mulligan, 1997) quanto afetado (Gabrieli et al., 1999; Mulligan \& Hartman, 1996; Mulligan, 1998; SchmitterEdgecombe, 1999).

Em geral, as evidências revisadas apoiam a hipótese de seleção do distrator (Mulligan, 2003), que prediz que quando distratores (itens de TS) exigem respostas frequentes e são apresentados como alvo, o processo de selecionar o distrator provavelmente prejudica a codificação do alvo e, assim, o priming. Como mencionado antes, a base dessa hipótese é o central bottleneck model (Pashler, 1994), no qual a codificação da memória, a recuperação da memória e a seleção da resposta dependem do mesmo amodal central bottleneck model. Pashler (1994) supõe que esse gargalo ou filtro (bottleneck) é uma característica estrutural de uma região ou rede cerebral que limita quantos processos essa região/rede pode realizar ao mesmo tempo. Logo, os processos que dependem desse gargalo precisam ser realizados de forma serial, de modo que dois processos não 
podem ocupar o mesmo gargalo. Assim, selecionar uma resposta ao estímulo da TS enquanto itens alvos de memória são apresentados reduz a disponibilidade do gargalo para os processos de codificação que, por sua vez, reduz o priming.

A hipótese de seleção do distrator explica confortavelmente o efeito da $\mathrm{AD}$ em diferentes testes de priming. Por outro lado, alguns resultados não se ajustaram à hipótese. Um exemplo foi o caso do teste de decisão de objeto possível/impossível, em que priming foi inalterado com uma TS de alta frequência de resposta, apresentada simultaneamente ao alvo (Soldan et al., 2008). Esse resultado não contradiz a hipótese de seleção do distrator, mas indica que a sincronia com o alvo e a frequência de resposta (aspectos importantes no priming perceptual verbal e com objetos familiares) são menos importantes ao priming na decisão de objeto possível/impossível. Objetos impossíveis não são encontrados no cotidiano, então é possível que exijam um processamento diferenciado. Além disso, os testes de decisão lexical e verificação de categoria também se mostram resistentes aos efeitos da $\mathrm{AD}$ mesmo com TS mais demandantes de atenção.

$\mathrm{O}$ attentional boost effect, identificado em Spataro et al. (2013), demonstrou que a AD, que tipicamente exerce efeitos deletérios na memória, pode melhorar o desempenho no priming. A TS consistia em detectar o círculo vermelho quando ele aparecesse com o estímulo alvo de memória. De acordo com Swallow e Jiang (2010, 2011), o attentional boost effect é causado pela abertura de um canal (gate) atencional após a detecção de alvos infrequentes nessa TS, o que aumenta a codificação perceptual do estímulo alvo simultaneamente. Nessa visão (Zacks, Speer,
Swallow, Braver, \& Reynolds, 2007), o mecanismo de gatting é implementado por regiões subcorticais e está envolvido no alerta do observador a mudanças salientes no ambiente. Assim, quando ocorre uma modificação do estímulo (círculos tornam-se vermelhos), o mecanismo de gatting é ativado, resultando no aumento da atenção às propriedades perceptuais da imagem, bem como na atualização de suas representações internas. $\mathrm{O}$ gate atencional seria como um filtro à entrada sensorial, facilitando o processamento perceptual da informação codificada com os círculos vermelhos a serem detectados. $\mathrm{O}$ attentional boost effect foi encontrado em decisão lexical e completar fragmentos de palavra, mas não em classificação semântica (Spataro et al., 2013), sugerindo que esse efeito pode dissociar tarefas perceptuais e conceituais. É necessário testar a generalização dos resultados para outras tarefas de priming e secundárias, inclusive para verificar se o efeito resultou da combinação específica dos testes utilizados nesse estudo.

Em relação à fase de recuperação de memória implícita, destacam-se os resultados de Lozito e Mulligan (2010) de imunidade do priming independentemente do tipo de teste (perceptual vs. conceitual), contrariando a predição da TAP (Roediger, 1990). Ainda, priming não foi influenciado pela frequência de resposta (alta vs. baixa), sugerindo que a dificuldade da TS não foi um fator significativo, contrariando a hipótese de seleção do distrator. Assim, foi apoiada a hipótese de automaticidade da recuperação implícita no referido estudo. É importante notar, porém, que embora Lozito e Mulligan (2010) tenham utilizado TS sincrônicas ao alvo e com alta frequência de resposta, elas podem não ter sido suficientemente demandantes de atenção. Isso 
porque monitorar se cada dígito é par ou ímpar ou se a palavra tem uma ou duas sílabas dispõe de um acesso rápido a representações acerca do status do número e de palavra no léxico, uma vez que esse conhecimento já está automatizado. Como descrito antes, Prull (2013) classificou a tarefa de decisão de número par ou ímpar como menos demandante de atenção se comparado à tarefa de aritmética mental.

Em síntese, as evidências revisadas não corroboram a hipótese de automaticidade dos processos implícitos, conforme postulado na literatura, na fase de codificação. Embora o priming tenha sido afetado pela $\mathrm{AD}$, as evidências também foram inconsistentes com as predições da TAP, pois os efeitos da atenção não foram relacionados ao fato de as tarefas serem perceptuais ou conceituais. Alternativamente, a hipótese de seleção do distrator tem recebido mais apoio empírico, dado que os efeitos da atenção têm sido relacionados ao grau de demandas atencionais exigidas pela TS. Essas conclusões, contudo, não podem ser aplicadas à fase de recuperação, pois os estudos são escassos e eles têm corroborado a hipótese de automaticidade.

\section{Referências ${ }^{4}$}

*Baques, J., Saiz, D., \& Bowers, J. S. (2004). Effects of working memory load on longterm word priming. Memory, 12, 301-313. Doi:10.1080/09658210244000469.

Blaxton, T. A. (1992). Dissociations among memory measures in memory-impaired subjects: evidence for a processing account of memory. Memory and Cognition, 20, 549562. Doi:10.3758/BF03199587.

*Clarke, A. J. B., \& Butler, L. T. (2008). Dissociating word stem completion and cued recall as a function of divided attention at retrieval. Memory, 16, 763-772. Doi: 10.1080/09658210802261116.

*Clarys, D., Isingrini, M., \& Haerty, A. (2000). Effects of attentional load and ageing on word-stem and word-fragment implicit memory tests. European Journal of Cognitive Psychology, $\quad 12, \quad 395-412$. Doi:10.1080/09541440050114561.

Craik, F. I. M., Govoni, R., Naveh-Benjamin, M., \& Anderson, N. D. (1996). The effects of divided attention on encoding and retrieval processes in human memory. Journal of Experimental Psychology: General, 125, 159180.

Doi:10.1080/00221309.2014.1003029.

*Gabrieli, J. D. E., Vaidya, C. J., Stone, M., Francis, W. S., Thompson-Schill, S. L., Fleischman, D. A., Tinklenberg, J. R., Yesavage, J. A., \& Wilson, R. S. (1999). Convergent behavioral and neuropsychological evidence for a distinction between identification and production forms of repetition priming. Journal of Experimental Psychology: General, 128, 479-498. Doi:10.1037/0096-3445.128.4.479.

Ganor-Stern, D., Seamon, J. G., \& Carrasco, M. (1998). The role of attention and study time in explicit and implicit memory for unfamiliar visual stimuli. Memory \& Cognition, 26(6), 1187-1195. Doi:10.3758/BF03201194..

\footnotetext{
${ }^{4}$ As referências acompanhadas de asterisco $\left(^{*}\right)$ fizeram parte da revisão sistemática.
} 
Graf, P., \& Schacter, D. L. (1985). Implicit and explicit memory for new associations in normal and amnesic subjects. Journal of Experimental Psychology: Learning, Memory, and Cognition, 11, 501-518. Doi:10.1037/0278-7393.11.3.501.

Hamann, S. B. (1990). Level-of-processing effects in conceptually driven implicit tasks. Journal of Experimental Psychology: Learning, Memory and Cognition, 16, 970-977. Doi:10.1007/s11910-002-0039-9.

*Horton, K. D., Wilson, D. E., Vonk, J., Kirby, S. L., \& Nielsen, T. (2005). Measuring automatic retrieval: a comparison of implicit memory, process dissociation, and speeded response procedures. ActaPsychologica, 119(3), 235-263. Doi: 10.1016/j.actpsy.2005.01.004.

*Isingrini, M., Vazou, F., \& Leroy, P. (1995). Dissociation between implicit and explicit memory tests: effects of age and divided attention on category exemplar generation and cued recall. Memory and Cognition, 23, 462-467. Doi:10.3758/PBR.16.3.475.

Jacoby, L. L. (1991). A process dissociation framework: separating automatic from intentional uses of memory. Journal of Memory and Language, 30, 513-541. Doi:10.1016/0749-596x(91)90025-f.

*Kellogg, R. T., Newcombe, C., Kammer, C., \& Schmitt, K. (1996). Attention and direct and indirect memory tasks with short- and long-term probes. American Journal of Psychology, $\quad$ 109, 205-217. Doi:10.1037/0022-3514.53.

*Light, L. L., \& Prull, M. W. (1995). Aging, divided attention, and repetition priming.
Swiss Journal of Psychology, 54, 87-101. Doi:10.1016/j.actpsy.2013.03.010.

*Light, L. L., Prull, M. W., \& Kennison, R. F. (2000). Divided attention, aging, and priming in exemplar generation and category verification. Memory \& Cognition, 28, 856-872. Doi: 10.3758/BF03198421.

Logan, G. D. (1990). Repetition priming and automaticity: common underlying mechanisms?. Cognitive Psychology, 22, 1-35. Doi:10.1016/0010-0285(90)90002-L.

*Lozito, J. P., \& Mulligan, N. W. (2010). Exploring the role of attention during implicit memory retrieval. Journal of Memory and Language, $\quad 63, \quad 387-399 . \quad$ Doi: 10.3758/BF03193246.

*Mulligan, N. W. (1997). Attention and implicit memory: the effects of varying attentional load on conceptual priming. Memory and Cognition, $\quad 25, \quad 11-17$. Doi:10.1080/09658210444000368.

*Mulligan, N. W. (1998). The role of attention during encoding on implicit and explicit memory. Journal of Experimental Psychology: Learning, Memory and Cognition, 24, 27-47. Doi:10.1037/0278-7393.24.1.27.

*Mulligan, N. W. (2003). Effects of cross-modal and intra-modal division of attention on perceptual priming. Journal of Experimental Psychology: Learning, Memory and Cognition, 29, 262-276. Doi:10.1037/02787393.29.2.262.

*Mulligan, N. W., \& Hartman, M. (1996). Divided attention and indirect memory tests. Memory and Cognition, 24, 453-465. Doi:10.3758/BF03200934.

*Mulligan, N. W., \& Hornstein, S. L. (2000). Attention and perceptual priming in the 
perceptual identification task. Journal of Experimental Psychology: Learning, Memory and Cognition, 24, 27-47. Doi:10.1037/0278-7393.26.3.626.

*Mulligan, N. W., \& Peterson, D. (2008). Attention and implicit memory in the category verification and lexical decision tasks. Journal of Experimental Psychology: Learning, Memory, and Cognition, 34(3), 662679. Doi:10.1037/0278-7393.34.3.662.

*Mulligan, N. W., \& Stone, M. (1999). Attention and conceptual priming: limits on the effects of divided attention in the category-exemplar production task. Journal of Memory and Language, 41, 253-280. Doi:10.1006/jmla.1999.2648.

*Mulligan, N. W., Duke, M., \& Cooper, A. W. (2007). The effects of divided attention on auditory priming. Memory \& Cognition, 35(6), 1245-1254. Doi:10.1027/1618$3169 / \mathrm{a} 000192$.

*Parker, A., Dagnall, N., \& Munley, G. (2012). Encoding tasks dissociate the effects of divided attention on category-cued recall and category-exemplar generation. Experimental Psychology, 59(3), 124-131. Doi:10.1027/1618-3169/a000134.

*Parkin, A. J., \& Russo, R. (1990). Implicit and explicit memory and the automatic/effortful distinction. European Journal of Cognitive Psychology, 2, 71-80. Doi: 10.1080/09541449008406198.

*Parkin, A. J., Reid, T. K., \& Russo, R. (1990). On the differential nature of implicit and explicit memory. Memory and Cognition, 18, 507-514. Doi:10.3758/BF03198483.

Pashler, H. (1994). Dual-task interference in simple tasks: data and theory. Psychological
Bulletin, 116, 220-244. Doi:10.1037/00332909.116.2.220.

Pashler, H., \& Johnston, J. (1998). Attentional limitations in dual-task performance. In $\mathrm{H}$. Pashler (Ed.). Attention (pp.155-189). New York: Psychology Press.

*Prull, M. (2013). Attention and repetition priming in the verb generation task. ActaPsychologica, 143, 218-226. Doi:10.1016/j.actpsy.2013.03.010.

Roediger, H. L. (1990). Implicit memory: retention without remembering. American Psychologist, $\quad 45, \quad$ 1043-1056. Doi:10.1037/0278-7393.6.5.576.

*Russo, R., \& Parkin, A. J. (1993). Age differences in implicit memory: more apparent than real. Memory and Cognition, 21, 73-80. Doi:10.3758/BF03211166.

Salles, J. F., Machado, L. L., \& Janczura, G. A. (2011). Efeitos de priming semântico em tarefa de decisão lexical em crianças de $3^{a}$ série. Psicologia: Reflexão e Crítica, 24(3), 597608. Doi:10.1590/S010279722011000300021

Schacter, D. L. (1987). Implicit memory: history and current status. Journal of Experimental Psychology: Learning, Memory and Cognition, 13, 501-518. Doi:10.1006/ccog.1998.0378.

*Schmitter-Edgecombe, M. (1996a). Effects of divided attention on implicit and explicit memory performance following severe closed head injury. Neuropsychology, 10, 155167. Doi:10.1037/0894-4105.10.

*Schmitter-Edgecombe, M. (1996b). The effects of divided attention on implicit and explicit memory performance. Journal of the International Neuropsychological Society, 2, 111 125. Doi:10.1037/0894-4105.10.2.155. 
*Schmitter-Edgecombe, M. (1999). Effects of divided attention on perceptual and conceptual memory tests: an analysis using a process-dissociation approach. Memory \&Cognition, 27, 512-525. Doi:10.3758/s13421-014-0464-4.

Seger, C. A., Rabin, L. A., Desmond, J. E., \& Gabrieli, J. D. E. (1999). Verb generation priming involves conceptual implicit memory. Brain and Cognition, 41, 150-177. Doi:10.1016/j.actpsy.2013.03.010.

*Smith, M. E., \& Oscar-Berman, M. (1990). Repetition priming of words and pseudowords in divided attention and in amnesia. Journal of Experimental Psychology: Learning, Memory, and Cognition, 16, 10331042. Doi:10.1037/0278-7393.16.6.1033.

*Soldan, A., Mangels, J. A., \& Cooper, L. A. (2008). Effects of dividing attention during encoding on perceptual priming of unfamiliar visual objects. Memory, 16, 873895. Doi:10.1080/09658210802360595.

*Spataro, P., Mulligan, N., \& Rossi-Arnaud, C. (2010). Effects of divided attention in the word-fragment completion task with unique and multiple solutions. European Journal of Cognitive Psychology, 22, 1511-1516. Doi:10.1080/09541440802685979.

*Spataro, P., Mulligan, N., \& Rossi-Arnaud, C. (2011). Effects of divided attention in the word-fragment completion task with unique and multiple solutions. European Journal of Cognitive Psychology, 22, 18-45. Doi:10.1080/09541440802685979.

*Spataro, P., Mulligan, N., \& Rossi-Arnaud, C. (2013). Divided attention can enhance memory encoding: the attentional boost effect in implicit memory. Journal of
Experimental Psychology: Learning, Memory, and Cognition, 39(4), 1223-1231. Doi:10.1080/13506285.2014.987859.

Stevens, W. D., Wig, G. S, \& Schacter, D. L. (2008). Implicit memory and priming In J. H. Byrne (ed.). Concise learning and memory (pp. 65-86). London: Academic Press.

Tulving, E., \& Schacter, D. L. (1990). Priming and human memory systems. Science, 247, 301306. Doi:10.1126/science.2296719.

Zacks, J. M., Speer, N. K., Swallow, K. M., Braver, T. S., \& Reynolds, J. R. (2007). Event perception: a mind/brain perspective. Psychological Bulletin, 133, 273-293. Doi:10.1037/0033-2909.133.2.273.

Recebido em: 18/1/2017 Aprovado em: 16/7/2018 